\title{
Bedside Teaching and Rounding for Clinical Pharmacy Profession
}

\author{
Asim Ahmed Elnour* \\ Department of Pharmacology, College of Medicine, UAE University, United Arab Emirates
}

\begin{abstract}
Received: January 22, 2014, Accepted: August 22, 2014, Published: August 26, 2014
*Corresponding author: Asim Ahmed Elnour, Adjunct Clinical Associate Professor, Department of Pharmacology, College of Medicine, UAE University, United Arab Emirates, Tel: + 971-506-734096; E-mail: assahura1962@gmail.com
\end{abstract}

It has been well documented in literature that physician's bedside teaching and rounding are valued and effective means to teach medical/medications history and physical exam skills for medical students, interns and residents. However, the pharmacy curriculum, pharmacist training and professional pharmacy practice lacks such inimitable favorable opportunity.

It was shown that medical practitioners bedside teaching (briefing and debriefing) and rounding permits other skills such as professionalism, communication, clinical observation and patient education (counseling) to be assessed, evaluated and prototyped. This vital aspect of medical education deserves some attention from the pharmacy professionals, academia and researchers. It is very imperative to develop models of bedside teaching and rounding for the clinical pharmacy practice.

There are enormous benefits of the bedside teaching and rounding for the clinical pharmacy profession. The intensive discussions performed during the bedside teaching and rounding will strengthen the pharmacist-patient relation, support the clinical pharmacist role, ease the communications with medical team and validate the clinical pharmacist as first-line care giver. The life scenarios in bedside teaching and learning environment provide more opportunities for improved patient outcomes that are significantly witnessed by the rounding team during the patient's length of stay.

The impact of pharmacy bedside teaching and rounding on patient's health outcome, merit to be studied and investigated by the pharmaceutical stakeholders. Whether or not this form of practice have profound benefits to the future of clinical pharmacy practice remains to be validated with comparative and randomized studies to delineate the components, 'pros and cons' and models of clinical pharmacy bedside teaching for students, interns and residents.

The momentum warrants to survey the pharmacy professionals about their opinions, beliefs and satisfaction of bedside teaching and rounding. The pharmaceutical education needs to incorporate and adopt these valid clinical practices onto the pharmacy curriculum, pharmacy student training, pharmacy interns and pharmacy residency programs.

The current available evidence to support this trend needs further elaboration and research to support the philosophical background related to pharmacy profession and not extrapolation from the medical profession. The clinical pharmacist can lead this initiative tailored to the benefits of the pharmacy students, interns, residents, junior clinical pharmacists. This should not obviate the growing functional multidisciplinary teams and will not undermine the collaborative practice model.

We urge more involvement of clinical pharmacists in pharmacy bedside teaching and rounding and demand on more intensive research emphasizing its impact on patient humanistic outcome, quality of life, quality of care and clinical outcome. 